\title{
Zur Arbeit mit diesem Buch
}

Ein gutes rechtswissenschaftliches Lehrbuch sollte nicht nur Wissen vermitteln, sondern auch Erfahrungen teilen. Denn der anwendungsbezogene Zuschnitt der juristischen Staatsprüfungen macht es erforderlich, das erlernte Wissen in der Praxis des juristischen Gutachtens abbilden zu können. Eine gute Klausurdarstellung wird aber nur gelingen, wenn der oder die Schreibende ausreichend Erfahrung beim Schreiben von Klausuren gesammelt hat. Das vorliegende Lehrbuch macht es sich deshalb zur Aufgabe, nicht nur das zu beherrschende Wissen, sondern auch eine „best practice des Klausurschreibens“ $\mathrm{zu}$ vermitteln.

Anders als im Zivil- und Strafrecht sind Klausuren im Öffentlichen Recht von Anfang an prozessual eingekleidet. Die Studierenden werden also nicht nur an die Frage herangeführt, ob Ansprüche zwischen den Streitparteien bestehen, sondern auch an die in der Praxis zentrale Frage ihrer gerichtlichen Durchsetzbarkeit. Die Lösung von Klausuren im Verwaltungsrecht macht zu 90\% die Beantwortung der Frage erforderlich, ob eine Klage bzw. ein Antrag vor dem Verwaltungsgericht Aussicht auf Erfolg hat (näher zu den Prüfungskonstellationen $\S 1$ Rn. 35 ff.). Was könnte also näher liegen, als ein Lehrbuch entsprechend dieser Klausurpraxis zu strukturieren?

Die Darstellung der einzelnen Themen erfolgt dafür orientiert am Gutachten in der Klausur. Diese Darstellungsform folgt nicht der Idee eines Generalschemas, mit dem alle verwaltungsrechtlichen Fälle erfasst werden könnten (zu Recht kritisch Schwerdtfeger/Schwerdtfeger, Öffentliches Recht in der Fallbearbeitung, 15. Aufl. 2018, Rn. 18). Das materiell-rechtliche Wissen wird vielmehr innerhalb der Struktur der klausurrelevanten gutachterlichen Prüfung der Klage- und Antragsarten erläutert. Während rein materiell-rechtliche Darstellungen die Lesenden oftmals mit der Frage allein lassen, wie das Wissen denn im juristischen Gutachten dargestellt werden sollte, präsentiert das vorliegende Lehrbuch das relevante Wissen und die zu beherrschenden Streitstände innerhalb der Besonderheiten der gutachterlichen Darstellung.

Die Ausführungen führen zunächst in die Themen ein und erklären die grundlegenden Zusammenhänge. Für Examenskandidat`innen und für die Lösung von Hausarbeiten relevante Aspekte sind extra gekennzeichnet worden und werden auf wissenschaftlichem Niveau vertieft, um den Lesenden die für ein Lehrbuch typische wissenschaftliche Durchdringung einer Problematik zu erlauben. Wer nur einen ersten Überblick erhalten will, kann auf die Lektüre der Vertiefungen zum Examenswissen und Hausarbeitswissen zunächst verzichten. Auch Formulierungsvorschläge werden i.S.v. „best practice“ angeboten. Die Ausführungen sind darüber hinaus mit Beispielen angereichert. Frei im Internet

Ә OpenAccess. (C) 2019 Nikolas Eisentraut, publiziert von De Gruyter. (cc)BB-sA Dieses Werk ist lizenziert unter der Creative Commons Attribution-ShareAlike 4.0. International. 
verfügbare Urteile/Beschlüsse sind in der digitalen Version des Lehrbuchs verlinkt, sodass direkt auf die relevante Rechtsprechung zugegriffen werden kann. Zudem wird das Lehrbuch von einem Fallrepetitorium begleitet, sodass das mit dem Lehrbuch erlernte Wissen anhand großer Klausurfälle auch in der praktischen Anwendung erprobt werden kann.

Die Beiträge des Lehrbuchs sind über Randnummern miteinander vernetzt, sodass trotz der Mehrzahl an mitwirkenden Autor^innen ein in sich geschlossenes Lernsystem entsteht. Randnummernverweise ohne §-Angabe beziehen sich auf den jeweils aufgeschlagenen §. Da in späteren Teilen des Lehrbuchs immer häufiger auf bereits eingangs vertieft ausgeführtes Wissen verwiesen wird, empfiehlt es sich, dem System des Lehrbuchs zu folgen und sich zunächst mit der Eröffnung der gutachterlichen Prüfung ( $(1)$ vertraut zu machen, bevor sodann die Anfechtungsklage (§ 2) und daran anschließend die weiteren Klage- bzw. Antragsarten bearbeitet werden.

In dem Kapitel „Literaturüberblick“ finden sich allgemeine weiterführende Literaturhinweise zum Verwaltungsrecht, wobei die dort angeführte Literatur überwiegend kostenpflichtig und nicht offen lizenziert ist. Auch die von den Autor ${ }^{\star}$ innen bearbeiteten einzelnen Abschnitte schließen mit Literaturhinweisen zu den jeweils spezifischen Fragestellungen, mittels derer einzelne Aspekte noch vertieft werden können. 